\title{
Direct Position Determination of Noncircular Sources with Multiple Nested Arrays: Reduced Dimension Subspace Data Fusion
}

\author{
Yang Qian $\mathbb{D}^{1,2}$ Dalin Zhao, ${ }^{1,2,3}$ and Haowei Zeng ${ }^{1,2}$ \\ ${ }^{1}$ College of Electronic Information Engineering, Nanjing University of Aeronautics and Astronautics, Nanjing 211106, China \\ ${ }^{2}$ Key Laboratory of Dynamic Cognitive System of Electromagnetic Spectrum Space (Nanjing University of Aeronautics \\ and Astronautics), Ministry of Industry and Information Technology, Nanjing 211106, China \\ ${ }^{3}$ Nanjing Institute of Supervision \& Testing on Product Quality, Nanjing 210008, China
}

Correspondence should be addressed to Yang Qian; qianyang@nuaa.edu.cn

Received 25 March 2021; Revised 21 May 2021; Accepted 4 August 2021; Published 29 August 2021

Academic Editor: Guimei Zheng

Copyright (c) 2021 Yang Qian et al. This is an open access article distributed under the Creative Commons Attribution License, which permits unrestricted use, distribution, and reproduction in any medium, provided the original work is properly cited.

Direct position determination (DPD) of noncircular (NC) sources with multiple nested arrays (NA) is investigated in this paper. Noncircular sources are used to expand the dimension of the received signal matrix, so the number of identifiable information sources and the accuracy of direct position determination are improved. Furthermore, nested array increases spatial degree of freedom. In this paper, the high-dimensional search problem of noncircular sources is investigated. Therefore, we propose algorithm dimension reduction subspace data fusion (RD-SDF) to reduce complexity and increase positioning accuracy. Simulation results show that the proposed RD-SDF algorithm for multiple nested arrays with noncircular sources has improved positioning accuracy with higher spatial degree of freedom than SDF, Capon, and two-step algorithms with uniform linear array and circular sources (CS).

\section{Introduction}

Nowadays, there are many research directions in wireless positioning technology. Traditional two-step location determination methods such as angle of arrival (AOA) and time difference of arrival (TDOA) are extensively used in navigation, seismic exploration, and industrial production [1-3]. Two-step location determination method estimates location parameters by the time, angle, and frequency difference [4]. In the process of positioning, the more intermediate processing steps are experienced, the more information is lost, and the subsequent positioning accuracy will be affected [5]. In order to solve the problem of two-step positioning, in recent years, direct position determination technology has been proposed [6]. In 2004, Weiss firstly proposed direct position determination [7]. This method obtains the target location from the observation data directly and need not any additional intermediate parameters [8-10]. Due to the direct use of observational data, the target information is fully utilized, and the parameter estimation steps are effectively reduced. So the positioning accuracy of the location can be greatly improved [11, 12].

With the fast development of computer and communication technology in recent years, direct positioning technology is used more and more. At present, most of the research is using angle of the sources or time delay for DPD with single station or multiple stations [13]. The DPD algorithm for multiple arrays mostly uses uniform linear array, which leads to low positioning accuracy due to the restricted array aperture [14].

Nowadays, there have been few reports about DPD methods making use of a nested array. In 2010, Pal and Vaidyanathan proposed a nested array structure, in which the more typical nested array consisted of two-stage subarrays [15]. Nested arrays increase spatial degree of freedom, so the number of identifiable information sources and the accuracy of direct position determination are improved [16-21]. Noncircular signal is a kind of signal that has been studied 
a lot now. Amplitude modulation signal, binary phase shift keying, and pulse amplitude modulation signal are indispensable signal forms in the modern communication system, and they all belong to noncircular source types. Therefore, the research of the NC source location algorithm has important practical significance [11, 22-25].

In this paper, the NC characteristics of an emitter signal are used to expand the spatial information to obtain larger virtual array aperture and more recognizable sources. Nested arrays are introduced into observation stations to increase the spatial degree of freedom and more recognizable sources, and then, combined with spatial smoothing technology, the DPD algorithm in the form of SDF for NC sources is derived. However, the high-dimensional search caused by the NC phase greatly increases the complexity of the algorithm. Therefore, this paper introduces multiple nested arrays into observation stations and proposes reduced dimension subspace data fusion (RD-SDF) algorithm with noncircular sources $[26,27]$.

We list the main contributions as follows:

(1) We introduce noncircular sources into the direct location algorithm model. We use noncircular sources to expand the dimension of the received signal matrix, so the number of identifiable information sources and the accuracy of direct position determination are improved.

(2) We introduce multiple nested arrays into multiple observation stations and combine with spatial smoothing technology. Furthermore, we smooth sum array and difference array and splice together to form a longer virtual array which increases spatial degree of freedom.

(3) We reduce the search dimension to lower the computational complexity. Furthermore, we lower the complexity of the algorithm to make sure of the estimation accuracy.

The structure of this paper is as follows. In Section 2, we introduce a few concepts of nested arrays and noncircular sources for DPD. In Section 3, we depict the proposed RDSDF algorithm. In Section 4, we depict the performance analysis about the RD-SDF algorithm for nested arrays with noncircular sources. In Section 5, we simulate the proposed RDSDF algorithm for nested arrays with noncircular sources. Finally, Section 6 summarizes this paper.

Notations are as follows: $(\bullet)^{H}$ denotes conjugate transpose, $(\bullet)^{*}$ denotes the conjugate, and $(\bullet)^{\mathrm{T}}$ denotes transposition. The symbol $\operatorname{vec}(\bullet)$ denotes the received covariance matrix virtualization, and symbol $\otimes$ denotes Kronecker product. $E(\bullet)$ denotes the mathematical expectation, and $\mathrm{I}_{n}$ denotes an $n \times n$ identity matrix.

\section{Preliminaries}

In this section, we introduce a few notions of nested array and the model of DPD. Then, we introduce a noncircular source model and characteristic.

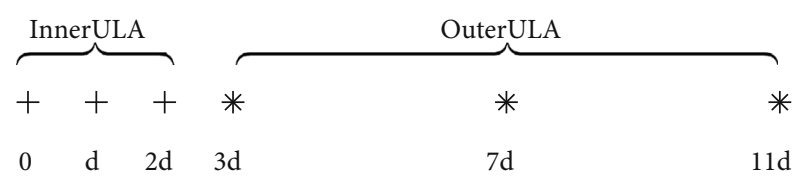

Figure 1: Two-level nested array.

2.1. Nested Array Model for Direct Positioning. We use a twostage nested array with $M=N_{1}+N_{2}$ sensors for direct position determination. The intensive uniform liner subarray has $N_{1}$ sensors in which interelement spacing is $d_{1}=d$. Another sparse one has $N_{2}$ sensors with $d_{2}=\left(N_{1}+1\right) d$, where $d=\lambda / 2$, and $\lambda$ is expressed as wavelength of the operating wave [20]. The array elements are set at $L^{\prime}=d L=\left\{l_{1}\right.$, $\left.l_{2}, \cdots, l_{N}\right\}$, and $L$ can be expressed as

$$
L=\left\{n_{1} \mid 0 \leq n_{1} \leq N_{1}-1\right\} \cup\left\{n_{2}\left(N_{1}+1\right)-1 \mid 1 \leq n_{2} \leq N_{2}\right\} .
$$

Figure 1 depicts a two-stage nested array, where $N_{1}=$ $N_{2}=3$. Figure 2 describes the sum coarray and difference coarray. In Figure 2, continuous virtual elements are locating from $-11 d$ to $11 d$ in the difference coarray with six physical sensors.

2.2. Multiple Nested Array Positioning Model. We use the positioning scenario as shown in Figure 3. Assuming that $Q$ uncorrelated far-field narrow-band noncircular sources are in the two-dimensional $X-Y$ plane [10]. There are $L$ twostage nested arrays located at $L$ stations. The target sources are located at $\mathrm{p}_{q}=\left[x_{q}, y_{q}\right]^{T}(q=1,2, \cdots Q)$ and $L$ stations are located at $\mathbf{u}_{l}=\left[x_{u l}, y_{u l}\right]^{T}(l=1,2, \cdots, L)$. Each observation station places $M$ array elements.

Assuming that the signal envelope changes slowly, the envelope of each signal is approximately equal when it arrives at the observation. The complex envelope of the $l$ th $(l=1,2, \cdots, L)$ observation corresponding to the $k$ th $(k=1,2,3 . \cdots K)$ sampling snapshot time can be expressed as [10].

$$
\mathrm{r}_{l}(k)=\sum_{q=1}^{Q} \mathrm{a}_{l}\left(\mathrm{p}_{q}\right) \mathrm{s}_{l, q}(k)+\mathrm{n}_{l}(k)
$$

where $s_{l, q}(k)$ denotes the signal waveform of the $q$ th source at the $k$ th sampling fast beat time of the $l$ th observation station, $\mathrm{a}_{l}\left(\mathrm{p}_{q}\right)$ denotes the direction vector, and $\mathrm{n}_{l}(k)$ denotes the noise vector of the antenna array of the $l$ th observation station. It is determined by the arrival direction of the signal $\theta_{l}\left(\mathrm{p}_{q}\right)[10]$.

$$
\theta_{l}\left(\mathrm{p}_{q}\right)=\arctan \frac{\mathrm{x}_{u l}(1)-\mathrm{p}_{q}(1)}{\mathrm{y}_{u l}(2)-\mathrm{p}_{q}(2)}
$$

$$
\mathrm{a}_{l}\left(\mathrm{p}_{q}\right)=\left[1, e^{-j 2 \pi d \sin \theta_{l}\left(\mathrm{p}_{q}\right)}, \cdots, e^{-j 2 \pi(M-1) d \sin \theta_{l}\left(\mathrm{p}_{q}\right)}\right]^{T} .
$$




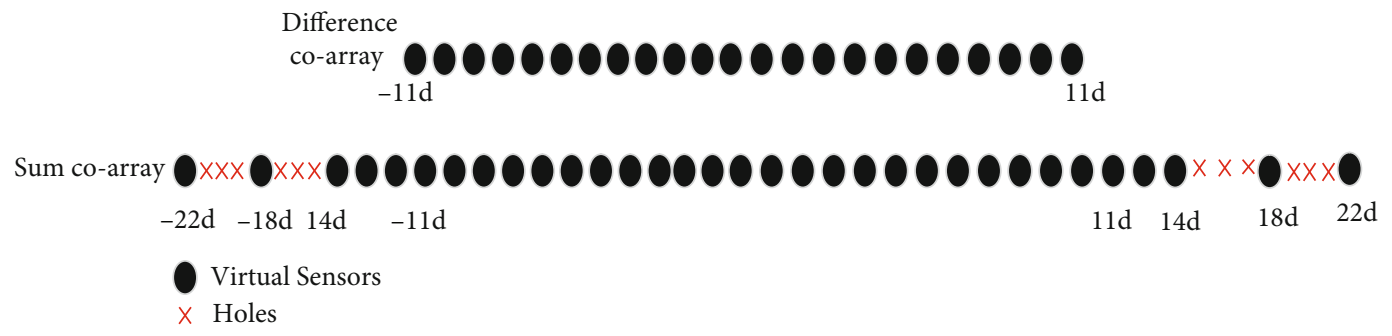

FIGURE 2: Difference coarray and sum coarray.

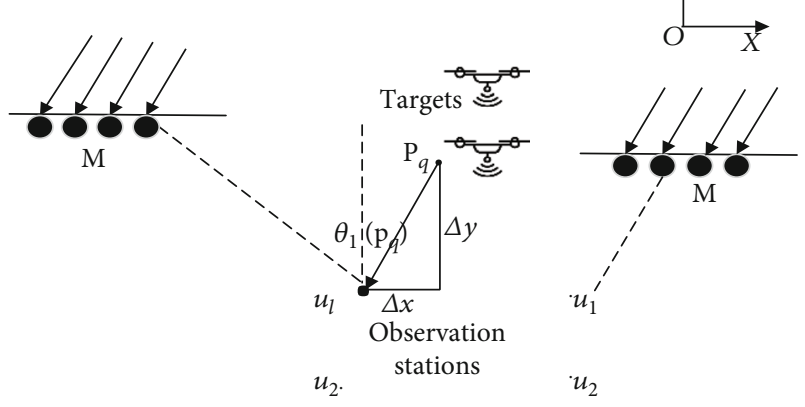

FIgURE 3: Multiple array combination positioning scene.

Equation (2) can be expressed as [10]

$$
\mathrm{r}_{l}(k)=\mathrm{A}_{l}(\mathrm{p}) \mathrm{s}_{l}(k)+\mathrm{n}_{l}(k)
$$

where

$$
\begin{gathered}
\mathbf{A}_{l}(\mathrm{p})=\left[\mathrm{a}_{l}\left(\mathrm{p}_{1}\right), \mathrm{a}_{l}\left(\mathrm{p}_{2}\right), \cdots, \mathrm{a}_{l}\left(\mathrm{p}_{\mathrm{Q}}\right)\right] \\
\mathrm{s}_{l}(k)=\left[\mathrm{s}_{l, 1}(k), \mathrm{s}_{l, 2}(k), \cdots, \mathrm{s}_{l, Q}(k)\right]^{T}, \\
\mathrm{p}=\left[\mathrm{p}_{1}^{T}, \mathrm{p}_{2}^{T}, \cdots, \mathrm{p}_{Q}^{T}\right]^{T}, \\
\mathrm{n}_{l}(k)=\left[\mathrm{n}_{l, 1}(k), \mathrm{n}_{l, 2}(k), \cdots, \mathrm{n}_{l, M}(k)\right]^{T}
\end{gathered}
$$

2.3. Noncircular Source Model. According to the second-order statistical properties, we can divide sources into circular sources and noncircular sources. For random vectors $s_{l}(k)$ of complex circular sources, covariance matrix $E\left[s_{l}(k) s_{l}^{H}(k)\right] \neq 0$, but ellipse covariance matrix $E\left[\mathrm{~s}_{l}(k) \mathrm{s}_{l}^{H}(k)\right]=0$. But for noncircular sources, neither is zero [6]. For noncircular sources, the covariance matrix and elliptic covariance matrix satisfy

$$
E\left[\mathrm{~s}_{l}(k) \mathrm{s}_{l}^{H}(k)\right]=\rho e^{j \varphi} E\left[\mathrm{~s}_{l}(k) \mathrm{s}_{l}^{T}(k)\right],
$$

where $\varphi$ is the noncircular phase and $\rho$ is the noncircular rate of the value in $0 \sim 1$.

The signal with a noncircular rate of 1 is called the maximum noncircular rate signal. For the sake of simplicity, this paper assumes that the noncircular rate is 1 . According to reference [11], noncircular sources can be expressed as

$$
\mathrm{s}(t)=\Phi \mathrm{s}^{0}(t)
$$

where

$$
\Phi=\left[\begin{array}{cccc}
e^{-j \varphi_{1}} & 0 & \cdots & 0 \\
0 & e^{-j \varphi_{2}} & \cdots & \cdot \\
\cdot & \cdot & \cdot & \cdot \\
0 & \cdot & 0 & e^{-j \varphi_{Q}}
\end{array}\right] \text {, }
$$

where $s^{0}(t)$ represents the real part of the signal.

According to equation (11),equation (5) can be rewritten as

$$
\mathrm{r}_{l}(k)=\mathrm{A}_{l}(\mathrm{p}) \Phi \mathrm{s}_{l}^{0}(k)+\mathrm{n}_{l}(k)
$$

where

$$
s_{l}^{0}(k)=\left[s_{l, 1}^{(0)}(k), s_{l, 2}^{(0)}(k), \cdots, s_{l, Q}^{(0)}(k)\right]^{T} .
$$

\section{The Proposed RD-SDF Algorithm}

In this section, we describe the proposed RD-SDF algorithm steps and describe the covariance vectorization signal and spatial smoothing technology in detail.

3.1. Covariance Vectorization Signal. According to the noncircular feature of the signal, we construct the direct position determination model by nested arrays. Combined with the idea of SDF, the cost function of the subspace data fusion algorithm with noncircular sources is obtained. Considering that the algorithm needs to search for high spectral peaks, this paper introduces the idea of dimension reduction, removes the noncircular phase search dimension, and finally gets the result reduced dimension subspace data fusion (RDSDF).

The vector of the received signal can be extended by using the characteristic that the elliptic covariance of the noncircular sources is not zero [11]. We construct the data model

$$
\mathrm{z}_{l}(k)=\left[\begin{array}{c}
\mathrm{r}_{1}(k) \\
\mathrm{r}_{1}{ }^{*}(k)
\end{array}\right]=\left[\begin{array}{c}
\mathrm{A}_{l}(\mathrm{p}) \mathrm{s}_{l}(k) \\
\mathrm{A}_{l}{ }^{*}(\mathrm{p}) \mathrm{s}_{l}^{*}(k)
\end{array}\right]+\left[\begin{array}{c}
\mathrm{n}_{l}(k) \\
\mathrm{n}_{l}^{*}(k)
\end{array}\right] .
$$

It can be obtained from equation (11)

$$
s_{l}^{*}(k)=\Phi^{*} s_{l}^{(0)}(k)=\Phi^{*} \Phi^{-1} s_{l}(k)=\left(\Phi^{*}\right)^{2} s_{l}(k) .
$$




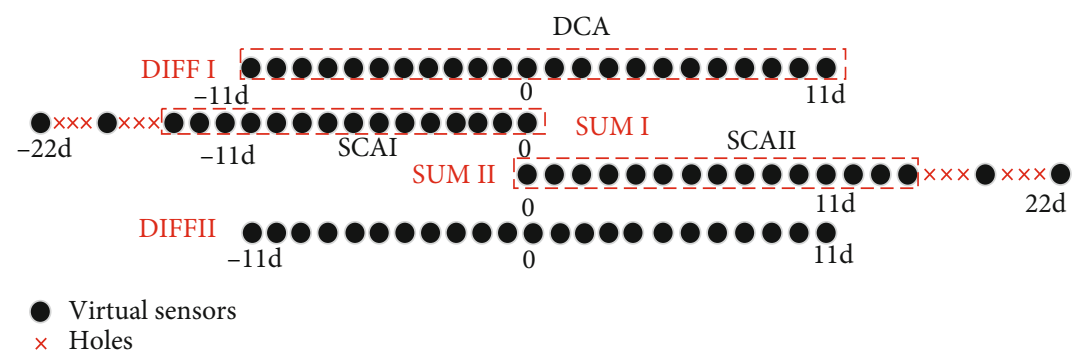

FIGURE 4: Structure diagram of sum difference array.

Then, equation (15) can be expressed as

$$
\begin{aligned}
\mathrm{z}_{l}(k) & =\left[\begin{array}{c}
\mathrm{A}_{l}(\mathrm{p}) \\
\mathrm{A}_{l}^{*}(\mathrm{p}) \Phi^{*} \Phi^{*}
\end{array}\right] \mathrm{s}_{l}(k)+\left[\begin{array}{c}
\mathrm{n}_{l}(k) \\
\mathrm{n}_{l}^{*}(k)
\end{array}\right] \\
& =\mathrm{B}_{l}(\mathrm{p}) \mathrm{s}_{l}(k)+\left[\begin{array}{c}
\mathrm{n}_{l}(k) \\
\mathrm{n}_{l}^{*}(k)
\end{array}\right],
\end{aligned}
$$

where

$$
\mathrm{B}_{l}(\mathrm{p})=\left[\begin{array}{c}
\mathrm{A}_{l}(\mathrm{p}) \\
\mathrm{A}_{l}(\mathrm{p}) \Phi^{*} \Phi^{*}
\end{array}\right]=\left[\mathrm{b}_{l}\left(\mathrm{p}_{1}\right), \mathrm{b}_{l}\left(\mathrm{p}_{2}\right), \cdots \mathrm{b}_{l}\left(\mathrm{p}_{Q}\right)\right],
$$

where

$$
\mathrm{b}_{l}\left(\mathrm{p}_{q}\right)=\left[\begin{array}{c}
\mathrm{a}_{l}(\mathrm{p}) \\
\mathrm{a}_{l}(\mathrm{p}) e^{j 2 \varphi_{q}}
\end{array}\right]
$$

The covariance matrix of the received signal is

$$
\mathrm{R}_{l}=\frac{1}{K} \sum_{k=1}^{K} \mathrm{Z}_{l}(k) \mathrm{Z}_{l}^{H}(k)=\sum_{i=1}^{q} \sigma_{l, q}^{2} \mathrm{~b}_{l}\left(\mathrm{p}_{q}\right) \mathrm{b}_{l}^{H}\left(\mathrm{p}_{q}\right)+\sigma_{n}^{2} \mathrm{I}
$$

where $\sigma_{l, q}^{2}$ represents the power of the $q$ th transmitted source at the $l$ th observation position and $\sigma_{n}^{2}$ represents noise power. In order to take advantage of characteristics of a nested matrix, we vectorize the covariance matrix [22]

$\mathrm{z}_{l}=\operatorname{vec}\left(\mathrm{R}_{l}\right)=\operatorname{vec} \sum_{i}^{D} \sigma_{l, q}^{2} \mathrm{~b}_{l}\left(\mathrm{p}_{q}\right) \mathrm{b}_{l}^{H}\left(\mathrm{p}_{q}\right)+\sigma_{n}^{2} \tilde{\mathrm{I}}=\mathrm{H}_{l}(\mathrm{p}) \mu+\sigma_{n}^{2} \tilde{\mathrm{I}}$, where $\mu$ is the signal power vector and

$$
\begin{gathered}
\mathrm{H}_{l}(\mathrm{p})=\left[\mathrm{b}_{l}^{*}\left(\mathrm{p}_{1}\right) \otimes \mathrm{b}_{l}\left(\mathrm{p}_{1}\right), \mathrm{b}_{l}^{*}\left(\mathrm{p}_{2}\right) \otimes \mathrm{b}_{l}\left(\mathrm{p}_{2}\right), \cdots, \mathrm{b}_{l}^{*}\left(\mathrm{p}_{\mathrm{Q}}\right) \otimes \mathrm{b}_{l}\left(\mathrm{p}_{\mathrm{Q}}\right)\right], \\
\tilde{\mathrm{I}}=\operatorname{vec}\left(\mathrm{I}_{M}\right),
\end{gathered}
$$

where

$$
\begin{aligned}
{\left[\mathrm{b}_{l}^{*}\left(\mathrm{p}_{l}\right) \otimes \mathrm{b}_{l}\left(\mathrm{p}_{l}\right)\right] } & =\left[\begin{array}{c}
\mathrm{a}_{l}\left(\mathrm{p}_{q}\right) \\
\mathrm{a}_{l}^{*}\left(\mathrm{p}_{q}\right) e^{j 2 \varphi_{q}}
\end{array}\right]^{*}\left[\begin{array}{c}
\mathrm{a}_{l}\left(\mathrm{p}_{q}\right) \\
\mathrm{a}_{l}^{*}\left(\mathrm{p}_{q}\right) e^{j 2 \varphi_{q}}
\end{array}\right] \\
& =\left[\begin{array}{c}
\mathrm{a}_{l}^{*}\left(\mathrm{p}_{q}\right) \otimes \mathrm{a}_{l}\left(\mathrm{p}_{q}\right) \\
\mathrm{a}_{l}^{*}\left(\mathrm{p}_{q}\right) \otimes \mathrm{a}_{l}^{*}\left(\mathrm{p}_{q}\right) e^{j 2 \varphi_{q}} \\
\mathrm{a}_{l}\left(\mathrm{p}_{q}\right) \otimes \mathrm{a}_{l}\left(\mathrm{p}_{q}\right) e^{-j 2 \varphi_{q}} \\
\mathrm{a}_{l}\left(\mathrm{p}_{q}\right) \otimes \mathrm{a}_{l}^{*}\left(\mathrm{p}_{q}\right)
\end{array}\right]=\left[\begin{array}{c}
d_{1} \\
d_{2} \\
d_{3} \\
d_{4}
\end{array}\right],
\end{aligned}
$$

where $d_{1}=\mathrm{a}_{l}^{*}\left(\mathrm{p}_{q}\right) \otimes \mathrm{a}_{l}\left(\mathrm{p}_{q}\right), d_{2}=\mathrm{a}_{l}^{*}\left(\mathrm{p}_{q}\right) \otimes \mathrm{a}_{l}^{*}\left(\mathrm{p}_{q}\right) e^{j 2 \varphi_{q}}, d_{3}=\mathrm{a}_{l}$ $\left(\mathrm{p}_{q}\right) \otimes \mathrm{a}_{l}\left(\mathrm{p}_{q}\right) e^{-j 2 \varphi_{q}}$, and $d_{4}=\mathrm{a}_{l}\left(\mathrm{p}_{q}\right) \otimes \mathrm{a}_{l}^{*}\left(\mathrm{p}_{q}\right)$. In the above equation $(24), \mathrm{a}_{l}^{*}\left(\mathrm{p}_{q}\right) \otimes \mathrm{a}_{l}\left(\mathrm{p}_{q}\right)$ and $\mathrm{a}_{l}\left(\mathrm{p}_{q}\right) \otimes \mathrm{a}_{l}^{*}\left(\mathrm{p}_{q}\right)$ can be used to generate difference coarray (Diff I and Diff II). While $\mathrm{a}_{l}^{*}($ $\left.\mathrm{p}_{q}\right) \otimes \mathrm{a}_{l}^{*}\left(\mathrm{p}_{q}\right) e^{j 2 \varphi_{q}}$ and $\mathrm{a}_{l}\left(\mathrm{p}_{q}\right) \otimes \mathrm{a}_{l}\left(\mathrm{p}_{q}\right) e^{-j 2 \varphi_{q}}$ can be used to generate the sum coarray (SUM I and SUM II).

The corresponding array structures of difference coarray 1 , sum coarray 1 , sum coarray 2 , and difference coarray 2 are shown in Figure 4 . The array elements of Diff I and Diff II are distributed as $\left[-\left(M_{1}-1\right) d,\left(M_{1}-1\right) d\right]$, where $M_{1}=N_{1} N_{2}+N_{2}$. The continuous array elements (SCA I and SCA II) of SUM I and SUM II are distributed as follows $\left[-\left(M_{2}-1\right) d, 0\right]$ and $\left[0,\left(M_{2}-1\right) d\right]$, where $M_{2}=$ $N_{1} N_{2}+N_{1}+N_{2}$. 


\subsection{Spatial Smoothing Technology and Noncircular Phase} Removal. In Figure 4, for continuous difference coarray DCA, we divide DCA into $R_{1}+1$ subarrays with equal length and equal distance; each subarray has $R_{1}+1$ elements.

According to references $[17,22], R_{1}=N_{1} N_{2}+N_{2}-1$, $R_{2}=0$, and $R_{3}=N_{1} N_{2}+N_{1}+N_{2}-1$. The received signal of the $i$ thi $\left(i=1,2, \cdots, R_{1}+1\right)$ subarray is $\mathrm{y}_{d i} \in C^{\left(R_{1}+1\right) \times 1}$ and can be expressed as

$$
\mathrm{y}_{d i}=\tilde{\mathrm{H}}_{d} \Psi^{i-1} \gamma+\sigma_{n}^{2} \tilde{\mathrm{u}}_{i}
$$

where $\mathrm{u}_{i}$ is the $i$ th element and is 1 , and the other elements are all vectors of $0 . \gamma$ is single snapshot correlation signal. $\mathrm{H}_{d}^{\sim}=\left[\mathrm{b}_{d}^{\sim}\left(\mathrm{p}_{1}\right), \mathrm{b}_{d}^{\sim}\left(\mathrm{p}_{2}\right), \cdots, \mathrm{b}_{d}^{\sim}\left(\mathrm{p}_{q}\right)\right]$ is the direction matrix of the first smooth subarray. Its $k$ th direction can be expressed as

$$
\tilde{\mathrm{b}}_{d}\left(\mathrm{p}_{q}\right)=\left[1, e^{-j \pi \sin \theta_{l}\left(\mathrm{p}_{q}\right)}, e^{-j 2 \pi \sin \theta_{l}\left(\mathrm{p}_{q}\right)}, \cdots, e^{-j R_{1} \pi \sin \theta_{l}\left(\mathrm{p}_{q}\right)}\right]^{T},
$$

$$
\Psi=\operatorname{diag}\left\{e^{j \pi \sin \theta_{l}\left(\mathrm{p}_{1}\right)}, e^{j \pi \sin \theta_{l}\left(\mathrm{p}_{2}\right)}, \cdots, e^{j \pi \sin \theta_{l}\left(\mathrm{p}_{Q}\right)}\right\} .
$$

The received signals of $R_{1}+1$ subarrays are combined together, and it can get the received signal matrix after spatial smoothing $\mathrm{Y}_{d} \in C^{\left(R_{1}+1\right) \times\left(R_{1}+1\right)}$.

$$
\begin{aligned}
\mathrm{Y}_{d} & =\left[\mathrm{y}_{d 1}, \mathrm{y}_{d 2}, \cdots, \mathrm{y}_{d\left(R_{1}+1\right)}\right] \\
& =\tilde{\mathrm{H}}_{d}\left[\gamma, \Psi \gamma, \cdots, \Psi^{\mathrm{R}_{1}} \gamma\right]+\sigma_{n}^{2} \mathrm{I}_{R_{1}+1} \\
& =\tilde{\mathrm{H}}_{d} \tilde{\mathrm{S}}+\sigma_{n}^{2} \mathrm{I}_{R_{1}+1},
\end{aligned}
$$

where $\mathrm{S}^{\sim}=\left[\gamma, \Psi \gamma, \cdots, \Psi^{R_{1}} \gamma\right]$ eliminates the equivalent signal matrix of correlation and $\mathrm{Y}_{d}$ can be expressed as the received signals of the first spatial smoothing subarray SS-Diff. The array element position range is $\left\langle 0, R_{1}\right\rangle$.

Then, the backward spatial smoothing is performed for SCA I and SCA II, respectively. In order to produce the same number of equivalent received signals, for continuous sum coarray, they are also divided into the $R_{1}+1$ subarray. After division, the $i$ th $i\left(i=1,2, \cdots, R_{1}+1\right)$ received signals of the SCA I and SCA II, respectively, are

$$
\mathrm{y}_{s i}^{-}=\tilde{\mathrm{H}}_{s}^{-} \Psi^{i-1} \gamma
$$

$$
\mathrm{y}_{s i}^{+}=\tilde{\mathrm{H}_{s}^{+}} \Psi^{i-1} \gamma
$$

where $\mathrm{H}_{s}^{-\sim}=\left[\mathrm{b}_{s}^{-\sim}\left(\mathrm{p}_{1}\right), \mathrm{b}_{s}^{-\sim}\left(\mathrm{p}_{2}\right), \cdots, \mathrm{b}_{s}^{-\sim}\left(\mathrm{p}_{q}\right)\right]$ is the direction matrix of the first spatial smoothing subarray of SCA I. The array element position of the SS-SCA I range is $\left\langle-\left(R_{3}-R_{1}\right), 0\right\rangle$ and its $q$ th direction vector can be expressed as

$\tilde{\mathrm{b}_{s}^{-}}\left(\mathrm{p}_{q}\right)=\left[e^{j\left(R 3-R_{1}\right) \pi \sin \theta_{l}\left(\mathrm{p}_{q}\right)} e^{j 2 \varphi_{q}}, \cdots, e^{j \pi \sin \theta_{l}\left(\mathrm{p}_{q}\right)} e^{j 2 \varphi_{q}}, e^{j 2 \varphi_{q}}\right]^{T}$,

where $\mathrm{H}_{s}^{+\sim}=\left[\mathrm{b}_{s}^{+\sim}\left(\mathrm{p}_{1}\right), \mathrm{b}_{s}^{+\sim}\left(\mathrm{p}_{2}\right), \cdots, \mathrm{b}_{s}^{+\sim}\left(\mathrm{p}_{q}\right)\right]$ is the first spatial smoothing subarray of SCA II. The array element position of the SS-SCA II range is $\left\langle R_{1}, R_{3}\right\rangle$ and its $q$ th direction vector can be expressed as

$\tilde{\mathrm{b}_{s}^{+}}\left(\mathrm{p}_{q}\right)=\left[e^{-j R_{1} \pi \sin \theta_{l}\left(\mathrm{p}_{q}\right)} e^{-j 2 \varphi_{q}}, e^{-j\left(R_{1}+1\right) \pi \sin \theta_{l}\left(\mathrm{p}_{q}\right)} e^{-j 2 \varphi_{q}}, \cdots, e^{-j R_{3} \pi \sin \theta_{l}\left(\mathrm{p}_{q}\right)} e^{-j 2 \varphi_{q}}\right]^{T}$.

Combining the received signals of all $R_{1}+1$ subarrays together, the equivalent received signal matrix of SS-SCA I and SS-SCA II can be obtained, respectively.

$$
\begin{aligned}
& \mathrm{Y}_{s}^{-}=\left[\mathrm{y}_{s 1}^{-}, \mathrm{y}_{s 2, \ldots,}^{-} \mathrm{y}_{s\left(R_{1}+1\right)}^{-}\right]=\tilde{\mathrm{H}}_{s}^{-} \tilde{\mathrm{S}}, \\
& \mathrm{Y}_{s}^{+}=\left[\mathrm{Y}_{s 1}^{+}, \mathrm{y}_{s 2, \ldots,}^{+} \mathrm{y}_{s\left(R_{1}+1\right)}^{+}\right]=\tilde{\mathrm{H}}_{s}^{+} \tilde{\mathrm{S}}
\end{aligned}
$$

After smoothing, the received signal is composed of $\mathrm{Y}_{s}^{-}, \mathrm{Y}_{d}, \mathrm{Y}_{s}^{+}$,

$$
\tilde{\mathrm{Y}}=\left[\begin{array}{c}
\mathrm{Y}_{s}^{-} \\
\mathrm{Y}_{d} \\
\mathrm{Y}_{s}^{+}
\end{array}\right]
$$

After smoothing, we splice SS-DCA, SS-SCA I, and SSSCA II together and connect three arrays end to end. A longer virtual array is formed. As shown in Figure 5, a longer virtual array is formed.

In order to lower the complexity and improve the practicability of the algorithm, this paper introduces the RD-SDF algorithm to reduce the search dimension and 


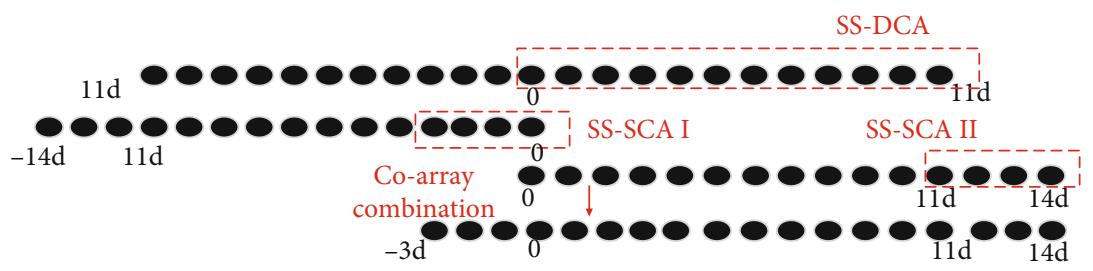

FIgURE 5: Array structure diagram corresponding to spatial smoothing.

remove the noncircular phase. After smoothing, equation (36) denotes the qth direction

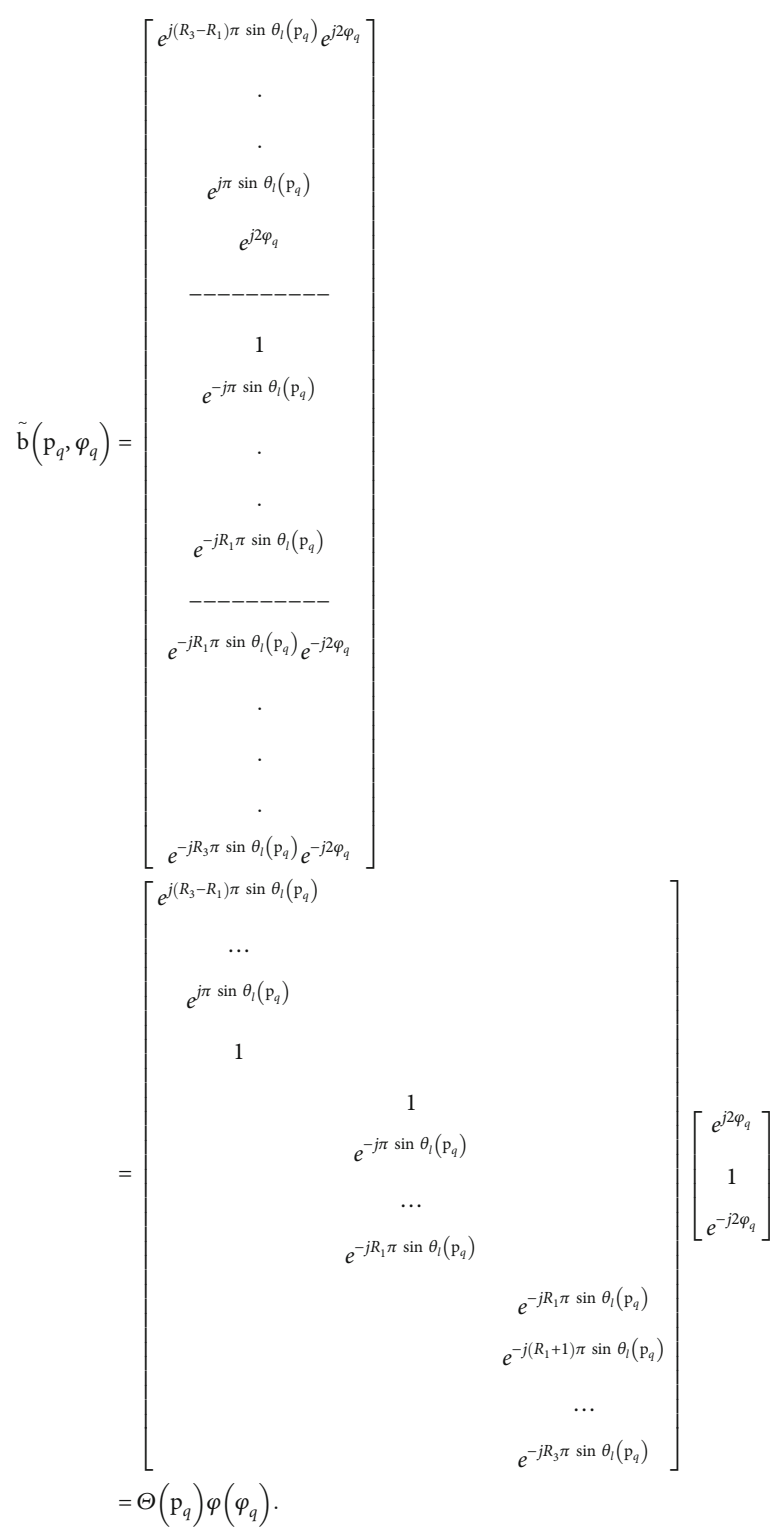

Therefore, separate the matrix $\varphi\left(\varphi_{q}\right)=\left[e^{j 2 \varphi_{q}} 1 e^{-j 2 \varphi_{q}}\right]^{T}$. $\Theta\left(\mathrm{p}_{q}\right)$ is another matrix after decomposition. We construct matrix $\mathrm{e}=\left[\begin{array}{lll}0 & 1 & 0\end{array}\right]^{T}$ to reduce dimension and remove the noncircular phase.
The cost function of noncircular direct location of nested array is

$$
f_{\mathrm{RD}-\mathrm{SDF}}(\mathrm{p})=\arg \max \sum_{l=1}^{L} \mathrm{e}^{H}\left(\Theta^{H}(\mathrm{p}) E_{l}^{n}\left(E_{l}^{n}\right)^{H} \Theta(\mathrm{p})\right) \mathrm{e} .
$$

3.3. The Steps of the Proposed RD-SDF Algorithm. We list several steps about RD-SDF algorithm.

Step1. Set up the noncircular sources and nested array model for DPD.

Step2. Generate sum coarray and difference coarray and adopt vector and spatial smoothing technology.

Step3. Calculate covariance matrix to obtain noise subspace $E_{l}^{n}$.

Step4. The noncircular phase is removed by dimension reduction method and the cost function is constructed $f_{\mathrm{RD}-\mathrm{SDF}}(\mathrm{p})$.

Step5. The target location search area is divided into several two-dimensional plane grids, and the cost function value corresponding to each grid point is calculated. The spectral peak searching value is the position estimation value $\left(x \wedge_{q}, y \wedge_{q}\right)$.

\section{Performance Analysis}

In this section, we analyze the performance of the RD-SDF algorithm, the obtained spatial degree of freedom, and the computational complexity of the RD-SDF algorithm and depict the advantages of the proposed RD-SDF algorithm.

4.1. Achievable DOFs. We define that $M$ represents the number of array elements, $N_{1}$ represents the number of intensive uniform liner subarray, and $N_{2}$ represents the number of sparse liner subarray. After smoothing, three smoothing subarrays are spliced together to form a longer virtual array. The DOF of the proposed algorithm after spatial smoothing is $N_{1} N_{2}+2 N_{1}+N_{2}$. Table 1 shows the DOF of the uniform linear array and nested array algorithm under circular and noncircular sources.

This paper emulates the degree of freedom of algorithm with different arrays for different sources. It can be seen from the Figure 6, the combination of noncircular sources and nested array improves the spatial degree of freedom and increases with the number of physical array elements. Therefore, it can identify more sources.

4.2. Complexity Analysis. We define that $M$ denotes array element numbers, $Q$ denotes source numbers, $L$ denotes observation station numbers, and $K$ denotes snapshot numbers. The $X$ direction is divided into $L_{x}$ equal parts, and $Y$ 
TABLE 1: DOF of different arrays with different sources.

\begin{tabular}{lc}
\hline Different arrays with different sources & DOF \\
\hline Circular sources for ULA & $M$ \\
NC sources for ULA & $2 M$ \\
Circular sources for nested array & $N_{1} N_{2}+N_{2}$ \\
NC sources for nested array & $N_{1} N_{2}+2 N_{1}+N_{2}$ \\
\hline
\end{tabular}

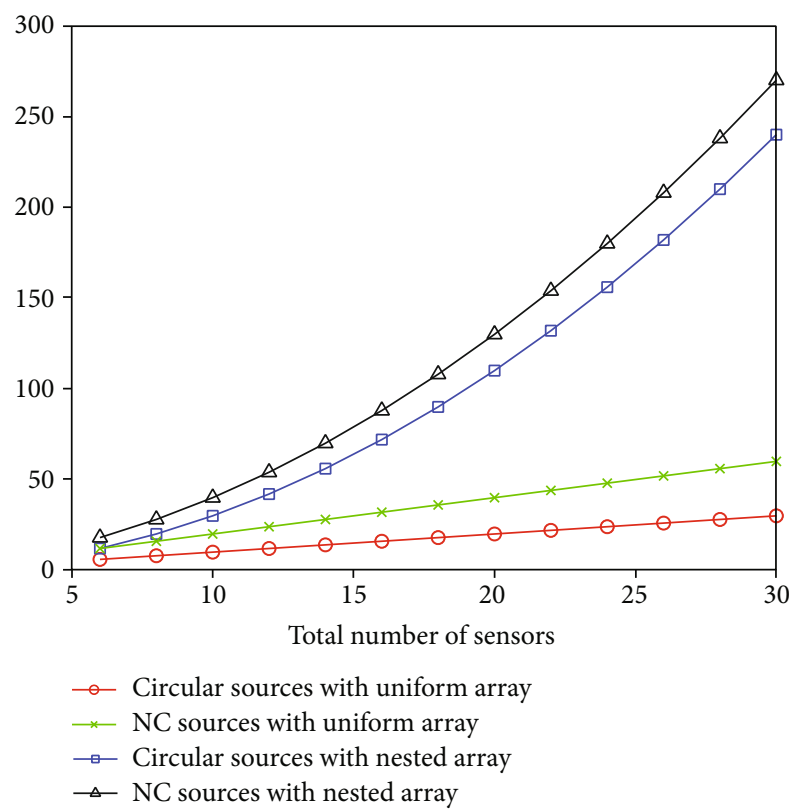

FIgURE 6: Comparison of DOF of different signals and arrays.

direction is divided into $L_{y}$ equal parts [10]. The noncircular phase is divided into $L_{\varphi}$. In this paper, the complexity of the algorithm before dimension reduction is mainly composed of covariance matrix calculation, eigenvalue decomposition, and search spectrum function calculation $[12,13]$.

The computational complexity of the RD-SDF algorithm contains several aspects: the calculation of covariance matrix of receiving sources $O\left(4 M^{2} L K\right)$ and the computational complexity of covariance after smoothing is $O[$ $\left.\left(N_{1} N_{2}+N_{2}+2 N_{1}\right)^{2}\left(N_{1} N_{2}+N_{2}\right) L\right]$. The eigenvalue decomposition of the covariance matrix of the receiving sources is $O\left[\left(N_{1} N_{2}+N_{2}+2 N_{1}\right)^{3} L\right]$, and the computational complexity of spectral peak value after dimension reduction is $O\left[L L_{x} L_{y}\right.$

$\left(3\left(N_{1} N_{2}+2 N_{1}+N_{2}\right)^{2}+9\left(N_{1} N_{2}+2 N_{1}+N_{2}\right)+\right.$ $\left.\left(N_{1} N_{2}+2 N_{1}+N_{2}\right)^{2}\left(N_{1} N_{2}+2 N_{1}+N_{2}-Q\right)+39\right]$.

Complexity before dimension reduction is

$$
\begin{aligned}
O & {\left[4 M^{2} L K+\left(N_{1} N_{2}+N_{2}+2 N_{1}\right)^{2}\left(N_{1} N_{2}+N_{2}\right) L\right.} \\
& +\left(N_{1} N_{2}+N_{2}+2 N_{1}\right)^{3} L+L L_{x} L_{y} L_{\varphi}\left(\left(N_{1} N_{2}\right.\right. \\
& \left.+2 N_{1}+N_{2}\right)^{2}+\left(N_{1} N_{2}+2 N_{1}+N_{2}\right) \\
& \left.\left.+\left(N_{1} N_{2}+2 N_{1}+N_{2}\right)^{2}\left(N_{1} N_{2}+2 N_{1}+N_{2}-Q\right)\right)\right] .
\end{aligned}
$$

Complexity after dimension reduction is

$$
\begin{aligned}
O & {\left[4 M^{2} L K+\left(N_{1} N_{2}+N_{2}+2 N_{1}\right)^{2}\left(N_{1} N_{2}+N_{2}\right) L\right.} \\
& +\left(N_{1} N_{2}+N_{2}+2 N_{1}\right)^{3} L+L L_{x} L_{y}\left(3 \left(N_{1} N_{2}\right.\right. \\
& \left.+2 N_{1}+N_{2}\right)^{2}+9\left(N_{1} N_{2}+2 N_{1}+N_{2}\right) \\
& \left.\left.\left.+\left(N_{1} N_{2}+2 N_{1}+N_{2}\right)^{2}\left(N_{1} N_{2}+2 N_{1}+N_{2}-Q\right)+39\right)\right)\right] .
\end{aligned}
$$

From the complexity, it can be seen that the complexity is significantly reduced after dimension reduction.

4.3. Advantages. We analyze advantages about the RD-SDF algorithm for nested arrays with noncircular sources.

(1) The proposed algorithm uses noncircular signal characteristics. Compared with the algorithm of circular sources and the uniform array, DOF has improved a lot.

(2) The high-dimensional search caused by the NC phase greatly increases the complexity of the algorithm. Based on this problem, we use the dimension reduction method to reduce complexity resulting from high-dimensional search. The computational complexity of the proposed RD-SDF algorithm is reduced.

(3) We compare the proposed algorithm with algorithms for uniform linear array and two-step positioning method, the positioning accuracy of the RD-SDF algorithm for a nested array with noncircular sources is greatly improved.

(4) We compare the proposed algorithm with algorithms for circular sources and two-step positioning method, the positioning accuracy of the proposed RD-SDF algorithm for nested arrays with noncircular sources is greatly improved.

\section{Simulation Results}

In this section, we simulate the proposed RD-SDF algorithm for nested arrays with noncircular sources, obtain the spectral peak map and location map, and compare the performance of the RD-SDF algorithm with other algorithms for DPD.

5.1. Simulations Results versus Proposed RD-SDF Algorithm. Multiple targets are $\mathrm{P}_{1}=[-800 \mathrm{~m}, 800 \mathrm{~m}], \mathrm{P}_{2}=[0 \mathrm{~m}, 500 \mathrm{~m}]$, and $\mathrm{P}_{3}=[800 \mathrm{~m}, 200 \mathrm{~m}]$. The noncircular phase is $(\pi / 6, \pi / 4$, $\pi / 3)$. There is a single target $P_{4}=[500 \mathrm{~m}, 300 \mathrm{~m}]$, and the noncircular phase is $\pi / 6$ correspondingly. Multiple nested arrays are located at multiple observation stations and observation stations are $U_{1}=[-1000 \mathrm{~m},-500 \mathrm{~m}], \mathrm{U}_{2}=[-500 \mathrm{~m},-500 \mathrm{~m}]$, $\mathrm{U}_{3}=[0 \mathrm{~m},-500 \mathrm{~m}], \mathrm{U}_{4}=[500 \mathrm{~m},-500 \mathrm{~m}]$, and $\mathrm{U}_{5}=[1000 \mathrm{~m},-$ $500 \mathrm{~m}]$. It can be seen from Figures 7 and 8 that the proposed algorithm can accurately locate a single target and multiple targets. Figure 9 shows the location map of the twodimensional target. 


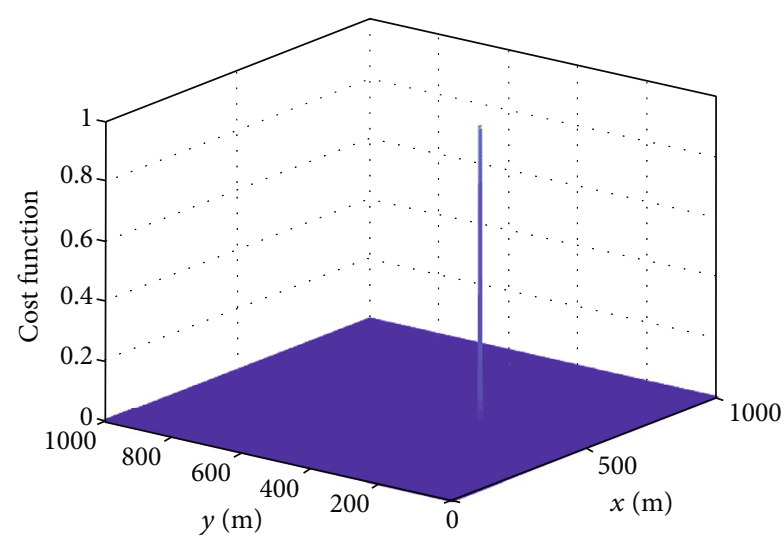

FIgURE 7: Single-target direct position.

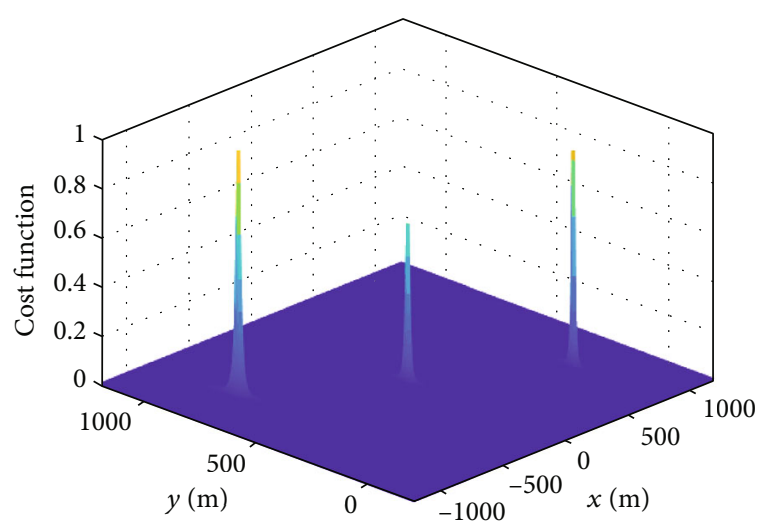

FIGURE 8: Multiple target direct position.

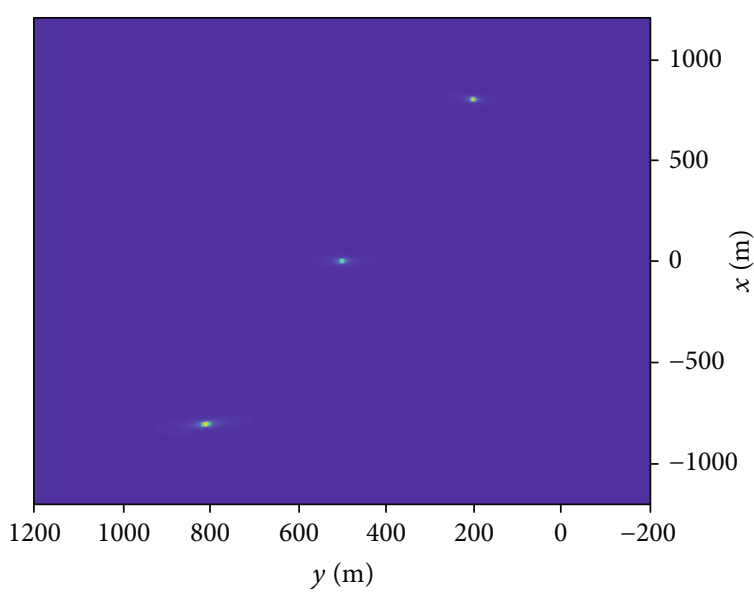

FIGURE 9: Direct location of 2D target location.

The estimation performance of the proposed RD-SDF algorithm is analyzed by calculating the root mean square error (RMSE), it can be denoted as [11].

$\operatorname{RMSE}=\frac{1}{Q} \sum_{q=1}^{Q} \sqrt{\frac{1}{\mathrm{MC}} \sum_{m c=1}^{\mathrm{MC}}\left[\left(x \wedge_{q, \mathrm{mc}}-x_{q}\right)^{2}+\left(y \wedge_{q, \mathrm{mc}}-y_{q}\right)^{2}\right]}$,

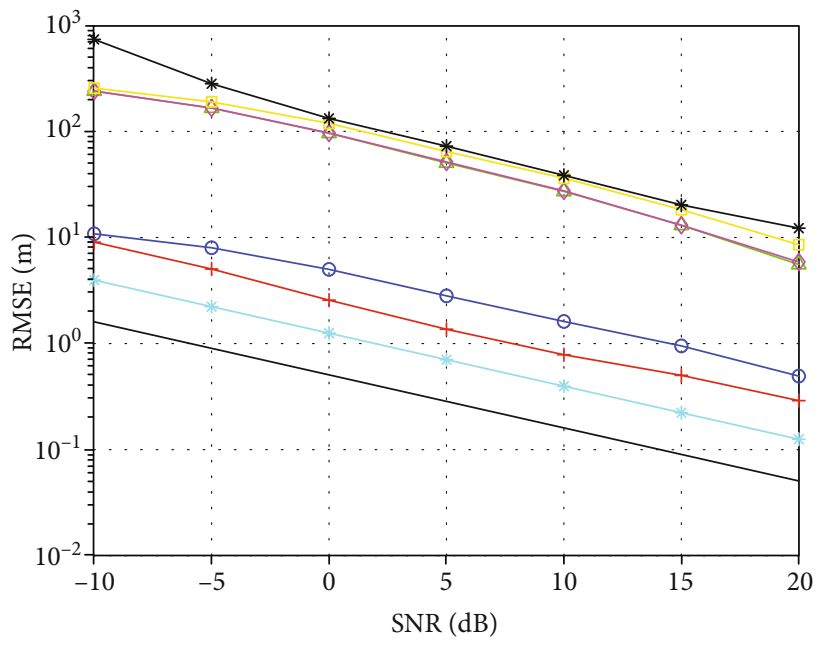

- RD-SDF for nested array with NC
- SDF for uniform array with CS
- PM for uniform array with CS
- CRB for uniform array with NC
- RD-SDF for uniform array with NC
- Capon for uniform array with CS
$\rightarrow-$ Two-step
- CRB for nested array with NC

FIgURE 10: Comparison of different algorithms for the nested array and uniform array.

where $\mathrm{MC}$ is the number of Monte Carlo (MC) simulation test, $Q$ is the number of target sources, $\left(x \wedge_{q, \mathrm{mc}}, y \wedge_{q, \mathrm{mc}}\right)$ represents the estimated value of the location of the $q$ th target in the mcth simulation experiment, and $\left(x_{q}, y_{q}\right)$ is the real value of the qth target.

5.2. RMSE Results versus Comparison of Different Algorithms with Different Arrays. The nested array element number is ( $\left.N_{1}, N_{2}\right)=(3,3)$. The snapshot number is 300 . Figure 10 shows the performance of the RD-SDF algorithm with different arrays for DPD. The performance of the RD-SDF algorithm for a nested array with noncircular sources is better than the algorithm of the RD-SDF algorithm for uniform array with noncircular sources. The performance of the RD-SDF algorithm for the nested array with noncircular sources is better than SDF, PM, and Capon algorithm [911] for uniform array with circular sources and the twostep localization algorithm. In Figure 10, CRB for the nested array with noncircular sources is better than CRB for a uniform array with noncircular sources.

5.3. RMSE Results versus Comparison of Different Algorithms with Circular Sources and Noncircular Sources. The nested array element number is $\left(N_{1}, N_{2}\right)=(3,3)$. The snapshot number is 300 . Figure 11 shows the comparison of algorithms with nested arrays under different signal types. The performance of the RD-SDF algorithm for noncircular sources is slightly better than the algorithm SDF for circular sources with nested array. Compared with the Capon algorithm and two-step positioning algorithm for circular sources with nested array, the proposed algorithm has better 


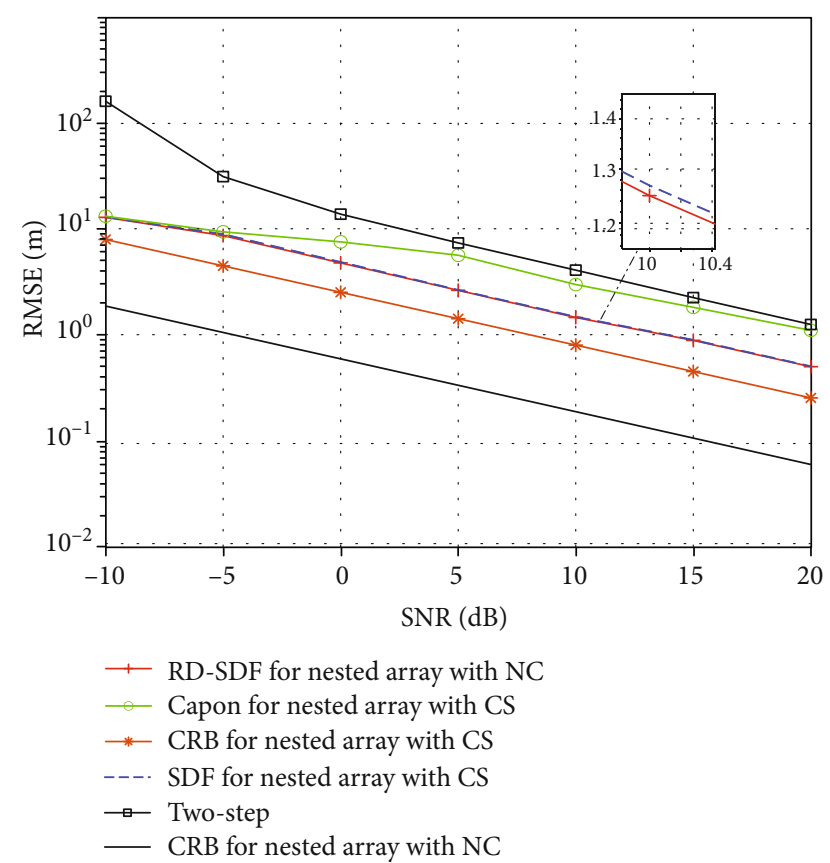

Figure 11: Algorithms comparison between circular sources and noncircular sources.

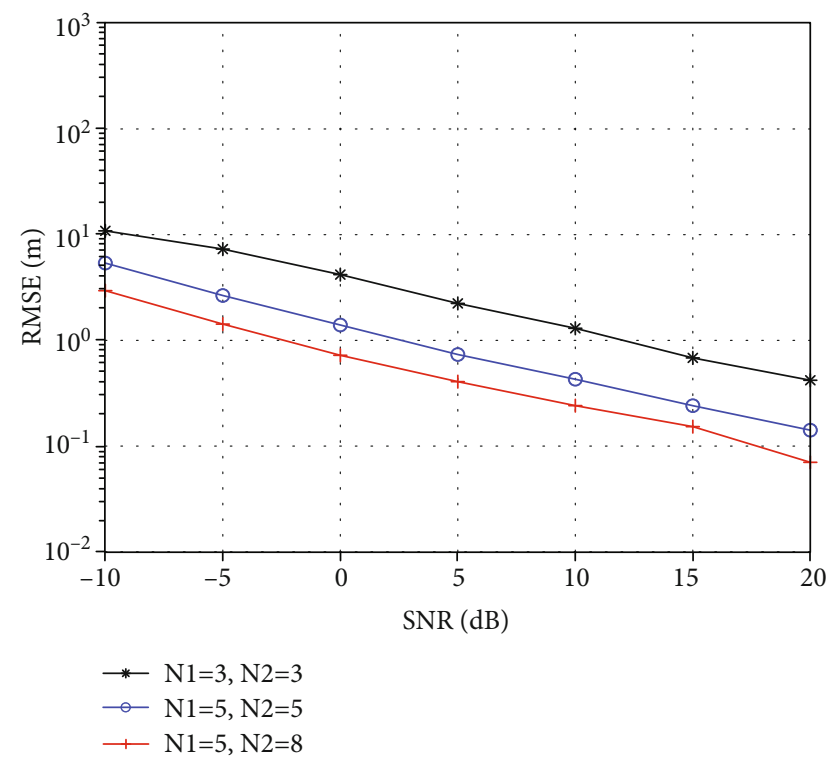

Figure 12: Comparison of different element numbers with RDSDF.

performance. CRB for nested array with noncircular sources is better than CRB for the nested array with circular sources.

5.4. RMSE Results versus Comparison of Different Array Element Numbers. The snapshot number is 300 . Figure 12 shows the performance of two subarrays of nested array with the number of elements $\left(N_{1}, N_{2}\right)=(3,3),(5,5),(5,8)$. When we improve the array element numbers, the performance of RD-SDF for nested array with noncircular sources will be relatively good.

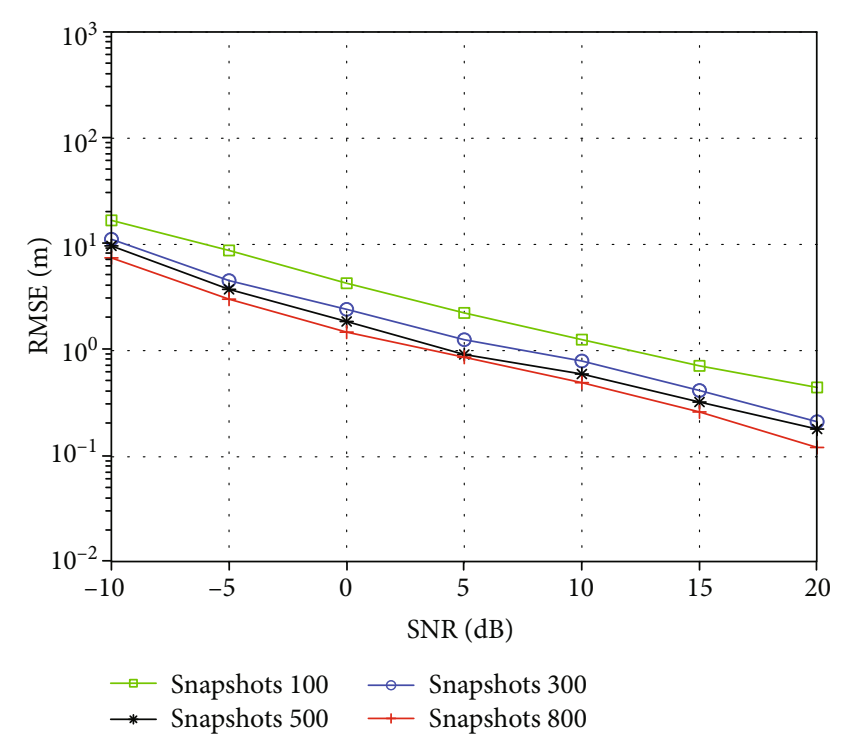

FIGURE 13: Comparison of RD-SDF under different snapshot numbers.

5.5. RMSE Results versus Comparison of Different Snapshot Numbers. This paper emulates the comparison of proposed algorithm by changing snapshot numbers. The number of snapshots was set to $100,300,500$, and 800 . The nested array element number is $\left(N_{1}, N_{2}\right)=(3,3)$. The performance of different snapshots is shown in Figure 13. With the increase of SNR, we can clearly see that to improve snapshot numbers, the performance of RD-SDF for nested arrays with noncircular sources will be better.

\section{Conclusion}

In order to improve direct positioning accuracy and spatial degree of freedom with uniform linear array and circular sources for DPD. This paper uses multiple nested arrays with noncircular sources for DPD. We use the RD-SDF algorithm to lower the computational complexity and increase positioning accuracy. The complexity of high-dimensional search caused by NC phase is eliminated by data reconstruction. Simulation results show that the proposed RD-SDF algorithm for nested array with noncircular sources has a prominent promotion in positioning accuracy with higher spatial degree of freedom than the SDF, Capon, and two-step algorithms with uniform linear array and circular sources.

\section{Data Availability}

The data used to support the findings of this study are included within the article.

\section{Conflicts of Interest}

The authors declare that they have no conflicts of interest. 


\section{Acknowledgments}

This work was supported by the National Key Research and Development Project Grant (2020YFB1807602).

\section{References}

[1] Y. Wang and Y. Wu, "An efficient semidefinite relaxation algorithm for moving source localization using TDOA and FDOA measurements," IEEE Communications Letters, vol. 21, no. 1, pp. 80-83, 2017.

[2] F. Wen, Q. Wan, and L. Y. Luo, "Time-difference-of-arrival estimation for noncircular signals using information theory," AEU-International Journal of Electronics and Communications, vol. 67, no. 3, pp. 242-245, 2013.

[3] Q. Liu, J. Xu, Z. Ding, and H. C. So, "Target localization with jammer removal using frequency diverse array," IEEE Transactions on Vehicular Technology, vol. 69, no. 10, pp. 1168511696, 2020.

[4] O. Bialer, D. Raphaeli, and A. J. Weiss, "Maximum-likelihood direct position estimation in dense multipath," IEEE Transactions on Vehicular Technology, vol. 62, no. 5, pp. 2069-2079, 2013.

[5] M. Oispuu and U. Nickel, "Direct detection and position determination of multiple sources with intermittent emission," IEEE Transactions on Signal Processing, vol. 90, pp. 30563064, 2010.

[6] J. Yin, D. Wang, and Y. Wu, "An efficient direct position determination method for multiple strictly noncircular sources," Sensors, vol. 18, no. 2, p. 324, 2018

[7] A. J. Weiss, "Direct position determination of narrowband radio frequency transmitters," IEEE Signal Processing Letters, vol. 11, no. 5, pp. 513-516, 2004.

[8] J. Wang, G. Ding, Q. Wu, L. Shen, and F. Song, "Spatial-temporal spectrum hole discovery: a hybrid spectrum sensing and geolocation database framework," Chinese Science Bulletin, vol. 59, no. 16, pp. 1896-1902, 2014.

[9] Y. Zhang, Y. Xu, B. Ba, D. M. Wang, and W. Geng, "Direct position determination of non-circular sources based on a Doppler-extended aperture with a moving coprime array," IEEE Access, vol. 6, pp. 6101-61021, 2018.

[10] Y. Qian, Z. Yang, and H. Zeng, "Direct position determination for augmented coprime arrays via weighted subspace data fusion method," Mathematical Problems in Engineering, vol. 2021, 10 pages, 2021.

[11] J. Yin, Y. Wu, and D. Wang, "Direct position determination of multiple noncircular sources with a moving array," Circuits, Systems, and Signal Processing, vol. 36, no. 10, pp. 405-4076, 2017.

[12] J. Li, L. Yang, F. Guo, and W. Jiang, "Coherent summation of multiple short-time signals for direct positioning of a wideband source based on delay and Doppler," Digital Signal Processing, vol. 48, pp. 58-70, 2016.

[13] Y. Zhang, B. Ba, D. Wang, W. Geng, and H. Xu, "Direct position determination of multiple non-circular sources with a moving coprime array," IEEE Sensors Journal, vol. 18, no. 5, 2018.

[14] J. Liu, Y. Zhang, Y. Lu, S. Ren, and S. Cao, "Augmented nested arrays with enhanced DOF and reduced mutual coupling," IEEE Transactions on Signal Processing, vol. 65, no. 21, pp. 5549-5563, 2017.
[15] P. Pal and P. P. Vaidyanathan, "Nested arrays: a novel approach to array processing with enhanced degrees of freedom," IEEE Transactions on Signal Processing, vol. 58, no. 8, pp. 4167-4181, 2010.

[16] J. Shi, G. Hu, X. Zhang, and H. Zhou, "Generalized nested array: optimization for degrees of freedom and mutual coupling," IEEE Communications Letters, vol. 22, no. 6, pp. 1208-1211, 2018.

[17] Y. Wang, W. Wu, X. Zhang, and Z. Zheng, "Transformed nested array designed for DOA estimation of non-circular signals: reduced sum-difference co-array redundancy perspective," IEEE Communications Letters, vol. 24, no. 6, pp. 12621265,2020

[18] C. Liu and P. P. Vaidyanathan, "Super nested arrays: linear sparse arrays with reduced mutual coupling-part I: fundamentals," IEEE Transactions on Signal Processing, vol. 64, no. 15, pp. 3997-4012, 2016.

[19] C. Liu and P. P. Vaidyanathan, "Super nested arrays: linear sparse arrays with reduced mutual coupling-part II: highorder extensions," IEEE Transactions on Signal Processing, vol. 64, no. 16, pp. 4203-4217, 2016.

[20] W. Si, Z. Peng, C. Hou, and F. Zeng, "Improved nested arrays with sum-difference coarray for DOA estimation," IEEE Sensors Journal, vol. 19, no. 16, pp. 6986-6997, 2019.

[21] J. Shi, F. Wen, and T. Liu, "Nested MIMO radar: coarrays, tensor modeling and angle estimation," IEEE Transactions on Aerospace and Electronic Systems, vol. 57, no. 1, pp. 573-585, 2021.

[22] Y. Wang, J. Shen, X. Zhang, Y. He, and X. Dai, "Non-circular signals for nested array: sum-difference co-array and DOA estimation algorithm," IET Radar, Sonar and Navigation, vol. 14, no. 1, pp. 27-35, 2020.

[23] X. Zhang, W. Chen, and W. Zheng, "Localization of near-field sources: a reduced-dimension MUSIC algorithm," IEEE Communications Letters, vol. 22, no. 7, pp. 1422-1425, 2018.

[24] P. Gupta and M. Agrawal, "Design and analysis of the sparse array for DOA estimation of noncircular signals," IEEE Transactions on Signal Processing, vol. 67, no. 2, pp. 460-473, 2019.

[25] B. Picinbono, "On circularity," IEEE Transactions on Signal Processing, vol. 42, no. 12, pp. 3473-3482, 1994.

[26] X. Zhang, L. Xu, L. Xu, and D. Xu, "Direction of departure (DOD) and direction of arrival (DOA) estimation in MIMO radar with reduced-dimension MUSIC," IEEE Communications Letters, vol. 14, no. 12, pp. 1161-1163, 2010.

[27] X. Zhang and D. Xu, "Angle estimation in bistatic MIMO radar using improved reduced dimension Capon algorithm," Journal of Systems Engineering and Electronics, vol. 1, pp. 8489, 2013. 